\title{
COVID-19 in Italy: The Point of View of the Italian Society of Pediatric Allergy and Immunology-COVID-19 Commission
}

\author{
Gian Luigi Marseglia, MD, ${ }^{1}$ Elena Chiappini, $\mathrm{PhD},{ }^{2}$ Ilaria Brambilla, $\mathrm{PhD},{ }^{1}$ Amelia Licari, $\mathrm{PhD},{ }^{1}$ \\ Maria Angela Tosca, $\mathrm{PhD}, \mathrm{PhD}^{3}$ and Giorgio Ciprandi, $\mathrm{MD}^{4}$
}

S EVERE ACUTE RESPIRATORY SYNDROME-CORONA VIRUS-2 (SARS-CoV-2) infection was first reported at the end of December 2019, and rapidly spread worldwide. Italy was the first European country sorely involved in the pandemic, with the first cluster occurring in South Lombardy. On March 11, 2020, the World Health Organization declared COVID-19 a global pandemic. On May 10, 2020, 4,024,737 subjects were infected worldwide, and 279,313 deaths reported. Italy ranks third in the number of cases $(218,000)$ after the United States and Spain, and fourth in the number of deaths (30,395) after the United States, the United Kingdom, and Brazil. However, as the pandemic continues to evolve, more pediatric cases have been identified. On May 30, 2020, 4,406 pediatric cases (1.9\% of the total COVID-19 patients) have been reported in Italy (www.epicentro.iss.it). There is a slight predominance of males $(51.4 \%)$. In the age segment $0-1$ year, 582 children $(13.2 \%)$ had COVID-19; in the 2-6 years old group, 767 cases $(17.4 \%)$; in the $7-17$, there were 3,057 cases (69.4). Totally, $105(2.4 \%)$ subjects were hospitalized: 31 $(29.5 \%)$ in the 0 - to 1-year age range, $17(16.2 \%)$ in the 2-6 years, and $57(54.3 \%)$ in the $7-17$ years age groups. Four children (one male and three females) died. All were $<9$ years of age and had comorbid conditions. Comorbidities included metabolic disease, cardiac malformations, and cancer.

Similar percentages were reported in the United States, China, and other European countries, which confirm that the pediatric population generally represents about $2 \%$ of all diagnosed cases. ${ }^{1}$ A systematic review included 18 studies and 1,065 children. The most frequently reported symptoms included cough, fever, pharyngitis, rhinorrhea, headaches, myalgia, rash, conjunctivitis, syncope, and gastrointestinal manifestations, such as vomiting, diarrhea, abdominal pain, and difficulty in feeding with varying percentages among studies. $^{2}$ Recently, a pediatric acute multisystemic inflammatory syndrome (PMIS) associated with COVID-19 was also reported. ${ }^{3}$ The precise classification is currently in process. This syndrome seems to share some clinical features with Kawasaki syndrome. ${ }^{4}$ Namely, both disorders are characterized by aberrant inflammatory response and re- sponsiveness to immunoglobulins, systemic corticosteroids, and anticytokine monoclonal antibodies.

In contrast, PMIS is characterized by multisystemic involvement, mainly concerning myocardial and/or gastrointestinal problems. In this regard, we reported a case concerning an adolescent with predominant obesity, severe diarrhea, and PMIS. ${ }^{5}$ Moreover, an increased incidence of Kawasaki syndrome associated with COVID-19 has been reported in Italy. ${ }^{6}$ Kawasaki syndrome is an acute and usually self-limiting vasculitis of the medium caliber vessels, which almost exclusively affects children. The possible link between PMIS and Kawasaki-like syndrome could be a secondary hemophagocytic lymphohistiocytosis reaction sustained by SARS-CoV-2 infection. ${ }^{7}$ A cytokine storm characterizes severe COVID-19 (Ref. $^{8}$ ). As proof of concept, anti-interleukin (IL)-1 treatment consistently induced a positive outcome in patients with severe COVID-19 lung disease. ${ }^{9}$ Similarly, it has been hypothesized that finger lesions (likened to "chilblains") observed in children with apparent increased frequency might be related to SAR-CoV2 infection, but there is no clarity on this issue, to date. ${ }^{10}$ SARS-CoV-2 also has a neuro-tropism, and the spectrum of neurological manifestations is vast. The most common neurosensorial symptom is anosmia due to viral penetration into the central nervous system through the olfactory bulb, without other nasal symptoms. ${ }^{11}$

Generally speaking, the current COVID-19 literature suggests that the clinical course is less relevant in children and adolescents in comparison with adults. Several hypotheses have been suggested, including the different expression of angiotensin-converting enzyme 2 (ACE2), the receptor of SARS-CoV-2. Age-dependent expression of ACE2 in nasal epithelium has been found to be less severe in children and adolescents as compared with adults. ${ }^{12}$ Moreover, comorbidities were considered risk factors for severe COVID-19. In this regard, children usually have fewer comorbidities than adults. Smoking was associated with increased expression of ACE2. Children also have a large thymic repertoire and sustained innate immunity, more

\footnotetext{
${ }^{1}$ Pediatric Clinic, Department of Pediatrics, Fondazione IRCCS Policlinico San Matteo, University of Pavia, Pavia, Italy.

${ }^{2}$ Infectious Disease Unit, Meyer Children's Hospital, Department of Science Health, University of Florence, Florence, Italy.

${ }^{3}$ Pediatric Allergy Center, Istituto Giannina Gaslini, Genoa, Italy.

${ }^{4}$ Allergy Clinic, Casa di Cura Villa Montallegro, Genoa, Italy.
} 


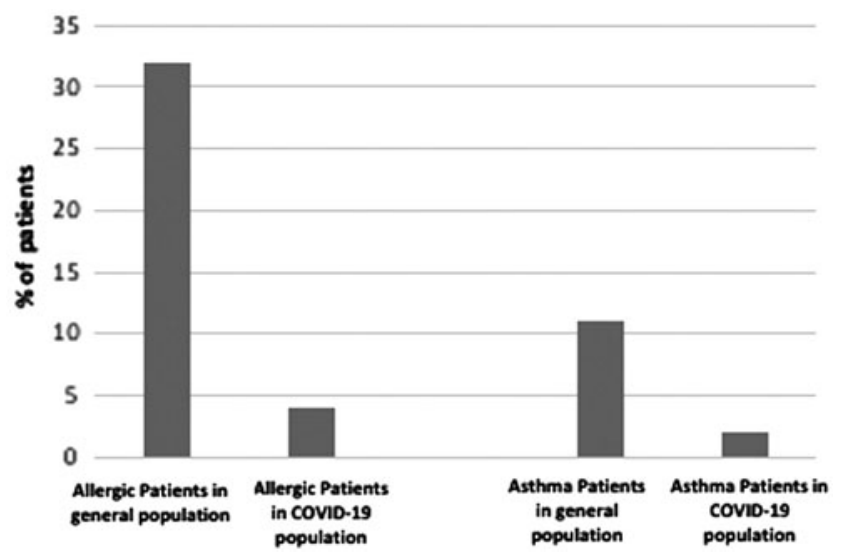

FIG. 1. Left part, prevalence of allergic patients in the general pediatric population and in the COVID-19 pediatric patients; right part, prevalence of asthmatic patients in the general pediatric population and the COVID-19 pediatric patients (Ref. ${ }^{14}$ ).

$\mathrm{T}$ and $\mathrm{B}$ regulatory lymphocytes than adults. Children experience several respiratory infections, including other Coronavirus, and the practice of vaccinations might constitute a robust immunological stimulus capable of activating the immune system and making it more efficient to contain pathogens in general. As a result, children could have a more protective immune response than adults. These concepts are consistent with our experience in two hub hospitals in Northern Italy: only one subject of 52 required high-intensity care ${ }^{13}$ Very interestingly, only two subjects (4\%) were allergic (food allergy and allergic rhinitis), and one (2\%) had asthma. ${ }^{14}$ These frequencies were lower than in the general population; namely, $32 \%$ of children suffer from allergies, and $11 \%$ have asthma in our country (Fig. 1). This outcome was consistent with preliminary studies that showed a very low prevalence of allergies and asthma in COVID-19 patients. ${ }^{15}$ In contrast, it has been reported that eosinopenia was very frequent $(81.2 \%)$ in patients dying from COVID-19 (Ref. ${ }^{16}$ ). Therefore, eosinopenia is considered a biomarker of poor prognosis and the best predictor to facilitate triage of adults with CODIV-19. It has been speculated that the reduced eosinophil count was not related to corticosteroid use but related to CD8 T cell depletion and eosinophil consumption caused by SARS-CoV-2. As eosinophilic cationic protein and eosinophil-derived neurotoxin can neutralize the virus, the eosinopenia could explain a higher SARS-CoV-2 load in turn, overconsume eosinophils. Eosinophils orchestrate the immune response to a respiratory virus, releasing cytotoxic proteins, increasing nitric oxide, producing type-1-associated cytokines, mainly IL-12 and interferon- $\gamma$, and recruiting CD8 T lymphocytes. Eosinophils clear viral load, so facilitating recovery from viral infections. As a consequence, it may be hypothesized that allergic patients, having eosinophilia, are less affected by COVID-19. We considered peripheral eosinophils and found low counts in COVD-19 children, whereas allergic children, without COVID-19, had significantly higher eosinophil count than COVID-19 patients. ${ }^{14}$ No eosinophils were identified in $5(12.5 \%)$ of the COVID positive cases. To further support the hypothesis that allergy might be "protective," a very recent study provided evidence that allergic sensitization was inversely related to ACE2 expression, and that natural allergen exposure significantly reduced ACE2 expression. ${ }^{17}$ It has also been recently noted that chronic respiratory diseases, including COPD and asthma, seem to be under-represented in the comorbidities of COVID-19 patients. $^{18}$

COVID-19 significantly affected the procedures that pediatricians perform in allergy settings or clinics. In this regard, the Italian Society of Pediatric Allergy and Immunology provided a Consensus Statement that contains a series of recommendations directed to the care of children and adolescents with allergic and immunological disorders concerning the use of nebulizers, spacers, and the maintenance of treatments. ${ }^{19}$ This document provides guidance for clinical examination, skin testing, spirometry, drug and food challenge, and allergen immunotherapy. ${ }^{19}$

In summary, COVID-19 seems to have a more favorable outcome in children and adolescents with allergy, as compared with adults. Attention to maintaining good control of asthma and allergy symptoms is recommended during the COVID-19 pandemic.

\section{Author Disclosure Statement}

The authors declare that the research was conducted in the absence of any commercial or financial relationships that could be construed as a potential conflict of interest.

\section{Funding Information}

No funding was received for this study.

\section{References}

1. Ludvigsson JF. Systematic review of COVID-19 in children shows milder cases and a better prognosis than adults. Acta Paediatr 2020; 109:1088-1095.

2. Castagnoli R, Votto M, Licari A, et al. Severe acute respiratory syndrome coronavirus 2 (SARS-CoV-2) infection in children and adolescents: a systematic review. JAMA Pediatr 2020 [Epub ahead of print]; DOI: 10.1001/jamapediatrics .2020 .1467$.

3. Shulman ST. Pediatric COVID-associated multisystemic inflammatory syndrome (PMIS). J Pediatr Infect Dis Soc 2020 [Epub ahead of print]; DOI: 10.1093/jpids/piaa062.

4. Viner RM, Whittaker E. Kawasaki-like disease: emerging complication during the COVID-19 pandemic. Lancet 2020 [Epub ahead of print]; DOI: 10.1016/S0140-6736(20)31129-6.

5. Brambilla I, Tosca MA, De Filippo M, et al. Special issues for COVID-19 in children and adolescents. Obesity (Silver Spring) 2020 [Epub ahead of print]; DOI: 10.1002/oby.22878.

6. Licciardi F, Pruccoli G, Denina M, et al. SARS-CoV-2induced Kawasaki-like hyperinflammatory syndrome: a novel COVID phenotype in children. Pediatrics 2020 [Epub ahead of print]; DOI: 10.1542/peds.2020-1711.

7. Verdoni L, Mazza A, Gervasoni A, et al. An outbreak of severe Kawasaki-like disease at the Italian Epicentre of the SARS-CoV-2 epidemic: an observational cohort study. Lancet 2020 [Epub ahead of print]; DOI: 10.1016/S01406736(20)31103-X.

8. Henderson LA, Canna SW, Schulert GS, et al. On the alert for cytokine storm: immunopathology in COVID-19. Arthritis Rheumatol 2020 [Epub ahead of print]; DOI: 10.1002/art.41285. 
9. Pontali E, Volpi S, Antonucci G, et al. Safety and efficacy of early high-dose IV Anakinra in severe COVID-19 lung disease. J Allergy Clin Immunol 2020 [Epub ahead of print]; DOI: 10.1016/j.jaci.2020.05.002.

10. Piccolo V, Bassi A. Acral findings during the COVID-19 outbreak: chilblain-like lesions should be preferred to acroischemic lesions. J Am Acad Dermatol 2020 [Epub ahead of print]; DOI: 10.1016/j.jaad.2020.05.077.

11. Gelardi M, Trecca E, Cassano M, et al. Smell and taste dysfunction during the COVID-19 outbreak: a preliminary report. Acta Biomed 2020 [Epub ahead of print]; DOI: 10.23750/abm.v91i2.9524.

12. Bunyavanich S, Do A, Vicencio A. Nasal gene expression of Angiotensin-converting enzyme 2 in children and adults. JAMA 2020 [Epub ahead of print]; DOI: 10.1001/jama .2020.8707.

13. Brambilla I, Castagnoli R, Caimmi S, et al. COVID-19 in the pediatric population admitted to a tertiary referral hospital in Northern Italy: preliminary clinical data. Pediatr Infect Dis J 2020 [Epub ahead of print]; DOI: 10.1097/ INF.0000000000002730.

14. Licari A, Votto M, Castagnoli R, et al. Allergy and asthma in children and adolescents during the COVID outbreak: what we know and how we could prevent allergy and asthma flares? Allergy 2020 [Epub ahead of print]; DOI: 10.1111/all.14369.

15. Zhang JJ, Dong X, Cao YY, et al. Clinical characteristics of 140 patients infected with SARS-CoV-2 in Wuhan, China. Allergy 2020 [Epub ahead of print]; DOI: 10.1111/all.14238.
16. Lindsley AW, Schwartz JT, Rothenberg ME. Eosinophil responses during COVID-19 infections and coronavirus vaccination. J Allergy Clin Immunol 2020; 146:1-7.

17. Jackson DJ, Busse WW, Bacharier LB, et al. Association of respiratory allergy, asthma, and expression of the SARSCoV-2 receptor, ACE2. J Allergy Clin Immunol 2020 [Epub ahead of print]; DOI: 10.1016/j.jaci.2020.04.009.

18. Halpin DM, Faner R, Sibila O, et al. Do chronic respiratory diseases or their treatment affect the risk of SARS-CoV-2 infection? Lancet Respir Med 2020 [Epub ahead of print]; DOI: 10.1016/S2213-2600(20)30167-3.

19. Cardinale F, Ciprandi G, Barberi S, et al. Consensus statement of the Italian Society of Pediatric Allergy and Immunology for the pragmatic management of children and adolescents with allergic or immunological diseases during the COVID-19 pandemic. Ital J Ped 2020 [Epub ahead of print]; DOI: 10.1186/s13052-020-00843-2.

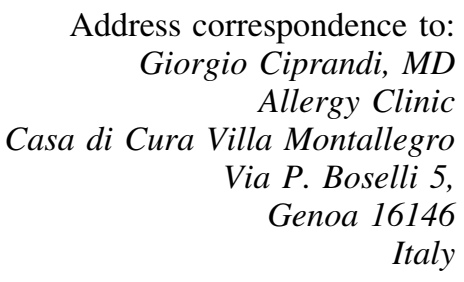

E-mail: gio.cip@libero.it

The findings, discussions, comments, or opinions expressed in "My Corner" are those of the author(s), and do not reflect those of the Editor-in-Chief, the journal publisher, or any of its affiliates. 